\title{
A Novel Centralization Method for Pipe Image Stitching
}

Salaheddin Hosseinzadeh, William Jackson, Dayi Zhang, Liam McDonald, Gordon Dobie, Graeme West, Charles MacLeod

\begin{abstract}
The creation of unwrapped stitched images of pipework internal surfaces is being increasingly used to augment routine visual inspection. A significant challenge to the creation of these stitched images is the need to estimate the pose and position of the camera for each frame, which is often alleviated through the use of a mechanical centralizer to ensure the camera is held in the center of the pipe. This article proposes a novel method for image centralization and pose estimation, which is particularly beneficial to circumstances where mechanical centralization is impractical. The approach involves post-inspection centralization of the captured video, by first estimating the probe's position relative to the pipe, using an integrated laser ring projector combined with the camera sensor, and then using this position to unwrap the image, so it produces an undistorted view of the pipe interior (equivalent to unwrapping a centralized view). These unwrapped images are then stacked to produce a stitched image of the pipe interior. In this paper pose estimation was successfully demonstrated to have a $90 \%$ confidence interval of $\pm 0.5 \mathrm{~mm}$ and $\pm 0.5^{\circ}$ in translation and rotation changes. This pose estimation is then used to create stitched images for both a visual test card image mounted inside a pipe and an aluminum pipe sample with artificial defects, in both cases demonstrating near equivalent results to those obtained using traditional mechanical centralization.

Index Terms - Remote visual inspection, Depth image-based rendering, photogrammetry, Image unwrapping, Post inspection centralization.
\end{abstract}

\section{Introduction}

OIL and gas, petrochemical, nuclear, and aerospace industries conduct regular assessment and examination of pipework to enable them to ensure safe operation, often mandated by safety regulations [1]. Remote Visual Inspection (RVI), where optical cameras are deployed to image key pipe surfaces is typically used where human access is constrained by either the small diameter of the pipework [2] or the environment in which the pipework resides.

In these scenarios, RVI is deployed by pushrods or robotic crawlers depending on the pipe internal bore diameter [3]. RVI requires minimal preparation of the pipework prior to inspection and, when compared to other available inspection methods, the data is much more intuitive to interpret. As a result, visual inspection is highly compatible with a relatively uncontrolled delivery method. However, centralization of the captured images is useful for both a human inspector, to aid localization during deployment and for recent approaches to converting inspection footage into stitched images of pipework internal surfaces.

\section{A. Robotically Deployed RVI}

Robotic crawlers are commonly used as a primary deployment platform for RVI of pipes typically larger than $150 \mathrm{~mm}$ in diameter by carrying the sensors and in some cases have an additional axis of motion to enable camera centralization [4]. Many researchers have adopted these crawler platforms such as

This work was supported in part by the Oil and Gas Innovation Centre (OGIC) in collaboration with Inspectahire Ltd (Aberdeen, UK).
Kwon et al. [5], which utilized a spring-loaded mechanism to adapt to the inner radius of the pipe $(80-100 \mathrm{~mm})$. This design provided an active method of camera centralization. In addition to the active centralization, the orientation and location of the camera were recorded using an accelerometer, a gyroscope and wheel encoders to aid in post-inspection camera pose estimation. Hansen et al. [6] designed a remote-controlled prototype crawler equipped with a fisheye camera, two laser diodes (a form of structured-light [7]), LEDs for illumination and a computer to inspect $400 \mathrm{~mm}$ diameter horizontal pipes. This allowed the creation of a sub-millimeter 3D model of the pipe using a combination of structure from motion (SfM) [8] and structured light. Duran et al. [9] mounted a camera and a laser ring profiler on a robotic manipulator. Coarse camera centralization was achieved by the camera on the manipulator. The laser-profiler was used for defect-type classification and not for the pose estimation. In addition, compared with the manually deployed system, the robotic manipulator can deploy the camera with a far finer degree of position control. Ting et al. [10] developed a crawler featuring an active stereo omnidirectional vision sensor, a series of LEDs, and a laserprofiler system for pipe RVI. In this work, the laser-profiler was used to provide visual cues and facilitate the unwrapping of pipe images. The authors in [11] proposed an autonomous underwater vehicle (AUV) for contactless RVI of aged pipelines with a diameter of $1 \mathrm{~m}$. The AUV had a single camera with ultrawide-angle lens (fisheye) and a laser-profiler. To enable autonomous navigation system a laser-profiler was mounted to the AUV, such that it projects a circular pattern on

(C) 20XX IEEE. Personal use of this material is permitted. Permission from IEEE must be obtained for all other uses, in any current or future media, including reprinting/republishing this material for advertising or promotional purposes, creating new collective works, for resale or redistribution to servers or lists, or reuse of any copyrighted component of this work in other works. 
the surface of the pipe within the field of view of the camera. The deformations of the laser-profiler were used to correct the relative path of the AUV. A lookup table of laser-profiler deformation was used to find the relative path. This mechanism, therefore, avoided any contact between AUV and the pipe wall. Unique lookup tables were required for each pipe radii and specific camera calibrations. Dehghan et al. [12] created a vision-based pose estimation technique for a pipe crawling robot. The system comprised of a collimated laser, a $45^{\circ}$ conic mirror, and a camera with a fisheye lens mounted on a robot. Instead of using a lookup table as in [11], authors in [12] used Nelder-Mead optimization [13] to estimate pose from the laser-profiler. However, due to the unknown nature of the objective function derivatives, the pose estimation success rate depended on the optimization's initial seed and may reduce to as low as $30 \%$.

\section{B. Push-Rod Deployed RVI}

Although these robotic crawlers presented in the previous section can facilitate RVI and in many cases the larger size and carrying capability of the crawler-based design provided the ability to utilize additional sensors such as [6] allowing the production of high-resolution surface profiles not possible otherwise, they are generally not as suitable for smaller bore diameter pipes $(<150 \mathrm{~mm})$. Also pipe systems with restricted access, or those with vertical sections to be traversed may be inaccessible to such platforms. In such cases, pushrod cameras with passive centralization systems are often used. For example, prior work carried out by West et al. [14] focused on the visual inspection of Advanced Gas-cooled Reactors (AGRs). In this case, a passive centralization system consisting of sprung guide wheels ensure the inspection tool is centralized in the channel. This passive mechanical centralizer eliminated the pose estimation requirement by keeping the probe approximately in the center of the tube at all times, while the camera assembly was deployed at a constant speed. A similar passive mechanical centralizer was adopted in [15] to keep the camera at the center of the pipe. The approach taken was to utilize 3D-printing to create a cylindrical spacer that matched the inner diameter of the pipe. A disadvantage of the centralizer-based approach is that it is generally only suitable for a set size of piping causing issues for example in sections with changing diameters, they may also snag on any protruding defects. Both drawbacks are avoided through the use of the authors post centralization method which utilizes a software approach to produce the same result as a physical centralizer.

\section{Automated RVI IMAGe Stitching}

An improvement to existing RVI is to perform a technique known as image stitching. The aim of this is to replace the traditional route of defect detection where recorded or live images are studied with a single image of the entirety of the pipework. This single image will consist of multiple frames which have been unwrapped and stitched together to provide a full view of the pipework under inspection.

There are many advantages of this method, as the image is scaled with distance the location of any detected defects is well known, this is in contrast to traditional RVI where the error of insertion distance is often observed to increase as it is generally measured from encoders which are known to suffer from slippage leading to mis-measurement.

When creating a stitched image of the inside of a pipe, two approaches are typically considered. Firstly, if the probe is centralized, images can be unwrapped using a transform based on the parameters of the camera and lens. In the case of a fisheye lens, this produces a slice representing a strip around the pipe. These slices are positioned one beside the next to form the stitched image. Secondly, in the case where the camera is not centralized, SfM [8] can be used to estimate the scene geometry and camera path to produce a textured $3 \mathrm{D}$ model of the pipe interior. Acosta et al. [2] compared several SfM algorithms for this application. Since SfM relies on feature matches between image pairs, it is highly sensitive to a surface finish which leads to inconsistent results [2]. Furthermore, the global optimization (bundle adjustment) required to estimate the full camera path is very computationally intensive and can take several hours to complete [8]. The quality of surfaces created from SfM solutions typically lacks the fidelity of unwrapped centralized images due to the relatively unbound nature of the SfM problem, examples detailing incomplete reconstructions of relatively sparse samples such as pipework can be found in [2]. This makes them less suitable for pipe inspections.

\section{Non-Centralized Automated RVI Image Stitching}

In this work, the aim is to extend the approach detailed in [14] (a $360^{\circ}$ panoramic image stitching technique) to the case of a non-centralized probe. The goal is to achieve equivalent robustness and high-quality results without the need for centralization. The authors make two assumptions to bound and essentially simplify the problem. The first assumption made is that that the images are of the inside of a perfectly round pipe and secondly that the probe has sensors that can estimate the position of the probe relative to the pipe.

The system, detailed in Section IV, consists of a camera fitted with a fisheye lens, laser projector and conical mirror with a $45^{\circ}$ reflecting angle. No centralizer is required as the postinspection centralization algorithm compensates for the impact of the non-centralized camera pose within the pipe. This algorithm is a combination of pose estimation and image unwrapping. The pose estimation and image unwrapping are implemented through a framework of MATLAB functions developed by the authors, it is noted that the optimization function used is part of the feature set developed by MathWorks [16]. The pose estimation utilizes the laser-profiler to calculate the probe position and orientation relative to the pipe. A novel algorithm is designed to eliminate the warping artifacts of noncentralized image unwrapping. The technique unwraps the images while maintaining consistent scale across the image set, achieved by incorporating the estimated camera poses into the process. This unwrapping is performed while compensating for the camera movement with regards to translation away from the center and rotations which cause the probe to become no longer co-axial with the pipe, by post-centralizing the probe such that 
images appear to be taken from a centralized camera. A simplified example of a grid image being unwrapped is illustrated in Fig. 1. Example (a) shows the expected image for a centralized probe, (b) shows the expected image for a noncentralized probe without applying the compensation algorithm presented in this paper and (c) shows the expected result by correcting for the pose.

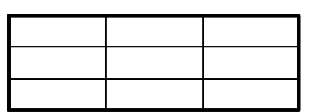

(a)

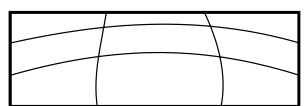

(b)

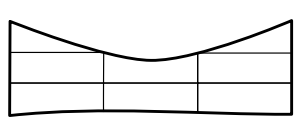

(c)

Fig. 1 Simplified cases of a grid like pattern being unwrapped, case (a) shows an image taken with the probe centralized, case (b) shows an expected result performing standard log polar unwrapping on a noncentralized probe without correcting for pose and case (c) shows the expected result when correcting for the pose as presented in this paper.

The unwrapped images of the pipe are then stitched together to create a single stitched image for long sections of pipework. The stitching process utilizes feature matching to estimate the distance between two images and combines them together to create a seamless join [17], similar to the image stitching process in [18]. Note that the process extracts unique features from the images and pairing of the features to calculate the distances, this may be problematic in featureless pipes. However, due to only using these features to calculate the distance in one dimension between the successive images for stitching and using the pose from the laser to aid in the process this issue is largely mitigated. The work presented is also targeted at visual inspections of pipework which have been in operation. Therefore, a certain number of features are expected on the images. Additionally, in unhealthy regions of the pipe, the surface finish tends to contain much more variation than clean healthy sections, and it is these sections that are the primary focus for the inspector.

The singular stitched image output from this process facilitates the visual inspection, defect detection and allows for chronological and comparative condition-monitoring. The remainder of the article is arranged as follows. Section IV pertains to the design of the probe and the implementation of the pose estimation and unwrapping algorithms. Results of empirical trials and accuracy analysis of the algorithms are provided in Section V. Section VI concludes with a discussion of results.

\section{SFMn AND PRobe Design}

The probe is comprised of a 5 MP camera (BFLY-PGE-50A2C) with a fisheye lens (Sunex PN DSL216), a laser-module, a $45^{\circ}$ conical mirror and illumination LEDs. The conical mirror and laser module are fixed in front of the camera and precisely aligned with the optical axis of the camera. The alignment procedure is further explained in [15]. The mirror is fixed at a distance of $\mathrm{d}=16 \mathrm{~mm}$ in front of the camera. The probe is depicted in Fig. 1 and has a length of $145 \mathrm{~mm}$ and a diameter of $45 \mathrm{~mm}$.

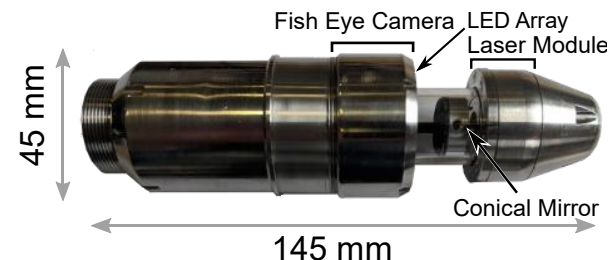

Fig. 2 The probe hardware used in this work with length and diameter dimensions indicated, the key subsystems are highlighted; camera module, laser module, LED array and the conical mirror.

The proposed visual inspection process in this paper has three steps:

1. Estimation of pose relative to the pipe

2. Unwrapping based on the relative pose

3. Stitching unwrapped sections

The main contribution of this article is the novel, laser-based, pose estimation where options for mechanical centralization are limited. Instead of using the conventional SfM, the proposed estimation utilizes a pair of images, captured by the probe (as shown in Fig. 2) to estimate the camera pose relative to the pipe. The first image of the pair is captured with the laser on and LEDs off, for pose estimation solely. This is to facilitate laser extraction as without additional illumination the laser line can be extracted more accurately. The second image is captured with the laser off and LEDs on to illuminate the pipe interior for the inspection. Similar to the commercial RVI approaches, the probe can be deployed manually as a standalone inspection tool or positioned by a robotic crawler as an integrated system [19], [20].

It is important to note than an assumption is made that the two sequence images are acquired with the same camera pose so that the laser image can be utilized to estimate the pose of the inspection image. This assumption is generally valid as the two images are captured within a suitably short time frame (77 $\mathrm{ms})$.

\section{A. Pose Estimation}

Pose estimation is enabled through the use of the laser module, the laser projects onto the conical mirror, which then reflects perpendicular to the camera's optical axis, forming a plane at the mirror location $(m)$. This is shown in Fig. 3. The reflection-plane is formulated as $\vec{n} \cdot(\overrightarrow{a-m})=0$, using a normal vector $(\vec{n}=m-o)$ and point $m$ on the reflection-plane, where $o$ is the camera location (assumed to be at the center of the origin), and $a$ is the location of any point that lies on the reflection-plane. Since the pipe is fixed, its geometry is defined in Eq. 1 as a cylinder (c) with an inner radius $r$ and length $l$ placed at the center of the origin in the direction of the coordinate system $z$ axis. The subscripts are notating $x, y$ and $z$ components of $c$. $\theta$ is swept from 0 to $2 \pi$.

$$
c_{x}=r \cos (\theta), c_{y}=r \sin (\theta), 0 \leq c_{z} \leq l, 0 \leq \theta \leq 2 \pi
$$

When $\vec{n}$ is aligned with the pipe's central axis $(\vec{n} \times \vec{z}=0)$, the projection of the reflection-plane onto the pipe forms a circular pattern with radius $r$. However, as the probe is deployed along the pipe, it may rotate for $\alpha$ and $\beta$ degrees 
about the $x$ and $y$ axes, respectively. Similarly, the probe may undergo a translation and deviate from the center of the pipe in the $x$ and $y$ directions for $\Delta x$ and $\Delta y \mathrm{~mm}$, respectively. The new location of the mirror $m^{*}$ and plane normal $\overrightarrow{n^{*}}$ as a result of this transformation can be described using the rotation (R) and translation matrixes (T) in Eq.2.

$$
\begin{gathered}
T=[\Delta x, \Delta y, 0], R=\left[\begin{array}{ccc}
\cos \beta & 0 & \sin \beta \\
\sin \alpha \sin \beta & \cos \alpha & -\sin \alpha \cos \beta \\
-\cos \alpha \sin \beta & \sin \alpha & \cos \alpha \cos \beta
\end{array}\right] \\
o^{*}=R \times \frac{o+T, m^{*}=R \times m+T}{\overrightarrow{n^{*}}=m^{*}-o^{*}}
\end{gathered}
$$

Therefore, in a more generalized case, where the probe is tilted $(\vec{n} \times \vec{z} \neq 0)$ and translated, the projection of the laserplane on the pipe wall $(p)$ (as shown as an outlined ellipse in Fig. 3) is determined by solving the intersection equation of the cylinder and the plane. This intersection is shown in Eq.3.

$$
\begin{gathered}
p_{x}=c_{x} \\
p_{y}=c_{y} \\
p_{z}=m_{z}^{*}-\frac{\left(n_{x}^{*}\left(x-m_{x}^{*}\right)+n_{y}^{*}\left(y-m_{y}^{*}\right)\right)}{n_{z}^{*}}
\end{gathered}
$$

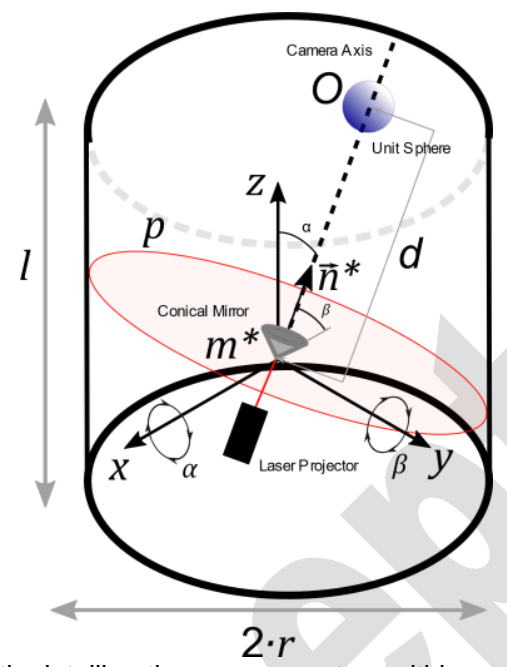

Fig. 3 Schematic detailing the camera system within a cylinder, $d$ is the distance from the center of the unit sphere as detailed in [21] to the point of reflection and origin of the laser plane $\mathrm{m}, \alpha$ is the rotation about the $x$ axis and $\beta$ the rotation around the $y$ axis.

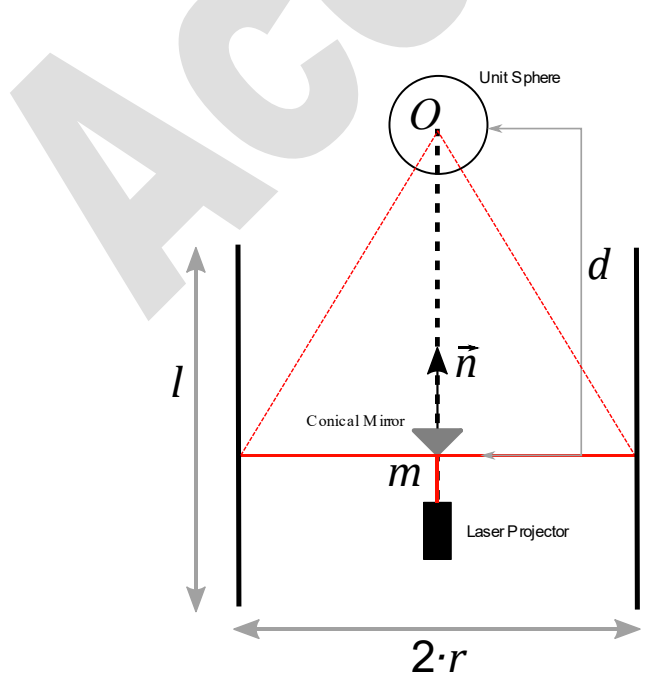

Fig. 4 Simplified two-dimensional schematic detailing the camera system centered within a pipe of diameter $2 r$. The dashed lines represent the rays of light observed by the camera at the intersection of the laser plane and pipe walls. The camera is represented by the omnidirectional camera model as detailed in [21]. $O$ is the camera origin located at the center of the unit sphere, $I$ is the length of the pipe, $m$ is the origin of the laser plane at the point of reflection on the conical mirror and $d$ is the distance between the camera origin and point of reflection

Since the conical mirror is aligned with the camera $\mathrm{z}$ axis, the rotation along the $\mathrm{z}$ axis does not impact the laser profile. Therefore, the pose estimation is performed to find the four unknown transformation parameters which describe the cameras position relative to the pipe center. These parameters consist of two rotations $(\alpha, \beta)$ and two translations $(\Delta x, \Delta y)$. To visualize the impact of these parameters on the image formation, $p$ is projected into the image plane, using Eq.4 [21], [22].

$$
P X(u, v, 1)=\boldsymbol{P} \cdot p(x, y, z, 1)
$$

Where $P X$ is the homogeneous coordinates of the laser ring pattern in the image plane and $\boldsymbol{P}$ is the projection matrix derived from the fisheye camera calibration [21]. The fisheye camera is calibrated by using the MATLAB built-in camera calibration toolbox [22]. The toolbox utilizes multiple checkerboard images captured by the camera to estimate the camera's extrinsic and intrinsic parameters [23]. Hence, Eq.5 combines Eq. 1 to 4 to express $P X$ as a function $(f)$ for a given pose.

$$
P X(u, v)=f(\alpha, \beta, \Delta x, \Delta y)
$$

An empirical example of such pattern $P X$ is depicted in Fig. 5 (red) and compared with a simulated centralized probe. To estimate the pose using the laser ring, first, the projected pattern on the pipe surface needs to be extracted. The intensity-based algorithm presented in [15] is used to extract the laser ring $P X(u, v)$ from the image.

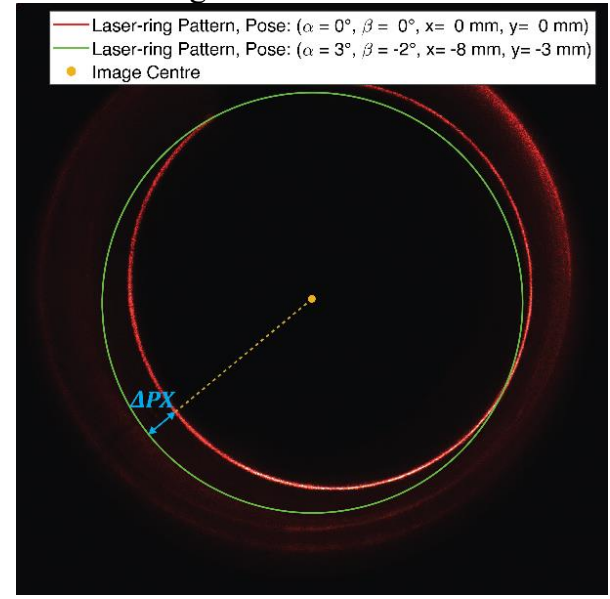

Fig. 5 Formation of laser ring pattern for a non-centralized (red) versus centralized (green) probe inside the $65 \mathrm{~mm}$ pipe. Green pattern is acquired from simulation. $\Delta \mathrm{PX}$ is the pixel difference between the centralized and non-centralized patterns. A small part of the laser ring (red) is missing due to the arm that holds the laser blocking the camera's field of view 
Once $P X$ is acquired, genetic algorithm optimization is employed to heuristically estimate the probe pose $(\tilde{\alpha}, \tilde{\beta}, \widetilde{\Delta} x, \widetilde{\Delta} y)$. The optimization finds the pose that minimizes the absolute distance between the estimated pattern $\widetilde{P X}($ Eq.5), and the observed laser ring pattern extracted from the image at all angles. To represent the movement restrictions of the probe inside the pipe and to avoid invalid poses and solutions, the optimization parameters (camera pose) are bounded. Assuming the probe diameter is negligible compared to the pipe diameter, probe translations are limited to $[-r, r]$. The probe rotations are limited to the maximum possible value that depends on the pipe internal diameter (inside a $65 \mathrm{~mm}$ internal diameter (ID) pipe the probe can rotate to $26.63^{\circ}$ ). In addition, two inequality constraints are considered for the optimization to assure that the camera and the conical mirror assembly are kept inside the pipe. The optimization and inequality constraints are formulated in Eq.6.

$$
\begin{gathered}
\underset{\widetilde{\alpha}, \widetilde{\beta}, \widetilde{\Delta} x, \widetilde{\Delta} y}{\operatorname{argmin}}(|P X-\widetilde{P X}|), \\
\left\{\widetilde{P X}=f(\tilde{\alpha}, \tilde{\beta}, \widetilde{\Delta} x, \widetilde{\Delta} y),|\widetilde{\Delta} x, \widetilde{\Delta} y|<r \text { and }|\widetilde{\alpha}, \tilde{\beta}|<\sin ^{-1}\left(\frac{2 r}{145}\right)\right\} \\
\text { Inequality constrains: }\left\{\begin{array}{l}
\widetilde{\Delta} x^{2}+\widetilde{\Delta} y^{2}<r^{2} \\
m_{x}^{2}+m_{y}^{2}<r^{2}
\end{array}\right.
\end{gathered}
$$

Note that the $145 \mathrm{~mm}$ in this equation is the probe length, as shown in Fig. 1. Physically, this process is analogous to locating the probe in different poses relative to the pipe until the best match to the observed pattern is found. Once the best match is found, the parameters of the optimization correspond to the camera pose.

\section{B. Sensitivity of Pose Estimation}

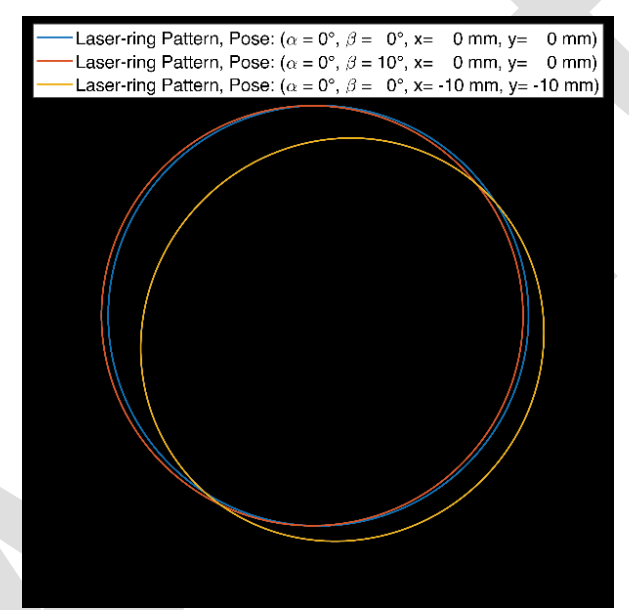

Fig. 6 Simulated laser ring patterns projected on a $65 \mathrm{~mm}$ pipe while the probe is positioned with various poses

Fig. 6 shows an example of the effect of translation and rotation of the probe relative to the pipe on the laser image in a $65 \mathrm{~mm}$ ID pipe. The sensitivity of the probe to translation and rotation can be determined by observing the maximum $\triangle \mathrm{PX}$ value. Where $\triangle \mathrm{PX}$ is calculated as the distance between each point of the ring for a simulated image taken with the probe centralized within the pipe and an image with rotation and translation applied to the probe $(\triangle \mathrm{PX}$ is shown graphically in Fig. 5). The simulation utilized the camera model obtained from the calibration of the physical probe used in this body of work. The maximum $\triangle \mathrm{PX}$ value for varying translation and rotation with respect to the $X$-axis of the probe within a $65 \mathrm{~mm}$ ID pipe is shown in Fig.7.

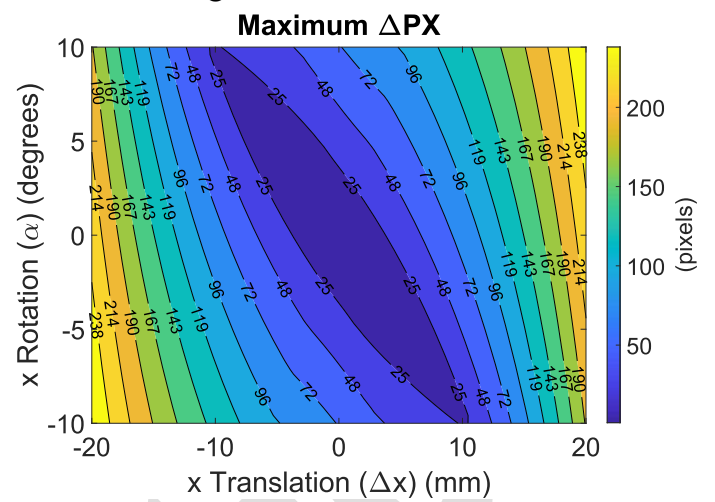

Fig. 7. Maximum $\triangle \mathrm{PX}$ pixel changes within a $65 \mathrm{~mm}$ ID pipe for varying rotational and translational camera pose changes

It is evident from Fig. 7 that the maximum $\triangle P X$ value is more sensitive to translation $(\Delta x, \Delta y)$ compared to rotation $(\alpha, \beta)$. It is also noted that the maximum sensitivity occurs when the rotation and translation are in the same direction, i.e. moving the laser and camera closer to the pipe wall and in contrast, the minimum sensitivity to change in pose is observed when they oppose each other.

The maximum value observed was a $\triangle P X$ value of 261 pixels for a $65 \mathrm{~mm}$ ID pipe. The simulation process was repeated on $50 \mathrm{~mm}$ and $80 \mathrm{~mm}$ ID pipes, the maximum $\triangle P X$ value for the smaller $50 \mathrm{~mm}$ ID pipe increased to 373 pixels and decreased to 196 pixels for the larger $80 \mathrm{~mm}$ ID pipe.

\section{Pose Estimate Performance Analysis}

To benchmark the pose estimation's accuracy and success rate, synthetic poses were analyzed for three different pipe diameters of 50,65 and $80 \mathrm{~mm}$ chosen to represent a range of diameters the system would likely operate in. These poses were generated randomly with a uniform distribution and their viability was checked using the inequality constrained in Eq.6. The rotation and translation parameters were bounded to $|\alpha, \beta|<\sin ^{-1}\left(\frac{2 r}{145}\right)$ and $|\Delta x, \Delta y|<r \mathrm{~mm}$, respectively. Using Eq. 5, an image of laser ring formation inside the pipe was generated for each camera pose, similar to that of Fig. 5. The laser ring was extracted, and the pose estimation was performed for each image. The error between the estimated poses and ground truth were then calculated for each pose component. The pose estimation success rate is defined as the capability of the algorithm to identify the correct pose within a certain margin of error $\left(T_{e}\right)$. Therefore, if the error of each estimated pose component is beyond $T_{e}$ then that estimation is deemed invalid. This indicates that the algorithm failed to identify the correct camera pose at that instance.

In the 300 trials undertaken on the $65 \mathrm{~mm}$ ID, $90.3 \%$ of the estimation were successful, where the error is below $T_{e}<0.5^{\circ}$ and $<0.5 \mathrm{~mm}$. The standard deviation and the absolute mean error of the success estimations are illustrated in Table 1. It is worth noting that the error observed with three different pipe diameters were similar, demonstrating the robustness of the method to the diameter of the pipe under inspection. 
Table 1. Pose estimation analysis results (65 mm ID pipe)

\begin{tabular}{|c|c|c|}
\cline { 2 - 3 } \multicolumn{1}{c|}{} & $\begin{array}{c}\text { Rotation Error } \\
\left({ }^{\circ}\right)\end{array}$ & $\begin{array}{c}\text { Translation Error } \\
(\mathbf{m m})\end{array}$ \\
\hline Standard Deviation & 0.0434 & 0.0122 \\
\hline Absolution Mean Error & 0.0081 & 0.0024 \\
\hline
\end{tabular}

\section{Image Unwrapping}

The unwrapping process is analogous to the transformation from polar to Cartesian coordinates, during which the images of a round pipe are flattened. The unwrapping techniques used in [10], [24] only work for a centralized probe, otherwise unwrapping leaves warping artefacts.

Therefore, a novel unwrapping algorithm based on depth image-based-rendering (DIBR) [25] and 3D ray tracing is used which can eliminate the aforementioned warping artefacts of a non-centralized probe, given the relative pose is provided and the pipe geometry is known. Both requirements are satisfied, as the pose is being estimated using the laser-profiler, and the pipe geometry is assumed cylindrical in Eq. 1. The unwrapping algorithm of non-centralized images (Fig. 8(a)) involves backprojecting [26] each pixel from the image plane to the surface of the pipe. However, to account for the camera deviation from the center of the pipe, first, the principal point of the camera is transformed with the transformation matrix using the estimated pose parameters. The next step is to create a point cloud of the pipe (Fig. 8(b)); hence, 3D ray tracing is used to back-project the image pixels to world coordinates and find their intersection with the surface of the pipe. Next, this point cloud is projected into a virtual camera that is located at the center of the pipe and aligned with its axis. This creates an image (Fig. 8(c)) equivalent to that of a centralized camera as if the probe was centralized. Finally, the centralized image is unwrapped (Fig. 8(d)) using log-polar unwrapping [24]. Through combining the pose estimation and unwrapping technique, a post-inspection camera centralization is achieved by estimating the pose and compensating for it. Note that the image unwrapping process is integrated into the authors MATLAB functions and is performed after the camera pose estimation.

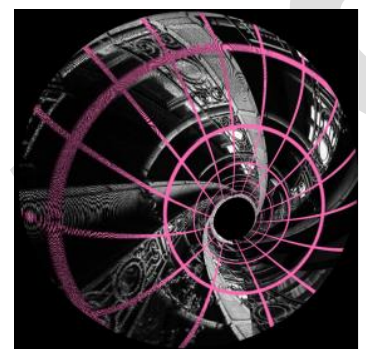

(a)

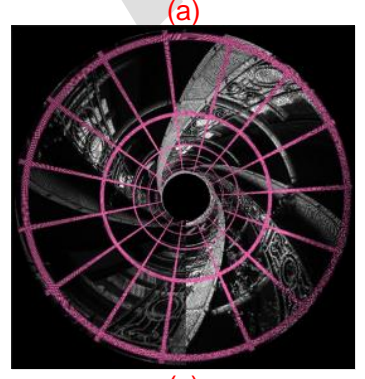

(c)

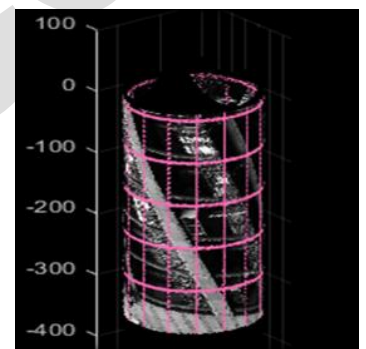

(b)

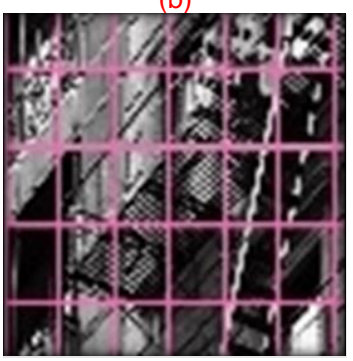

(d)
Fig. 8 Unwrapping process diagram of non-centralized images. (a) is the input non-centralized raw image. (b) is the point cloud of the pipe created from the pose observed and the input image (a), (c) is the postprocessed, centralized image using the point cloud (b). (d) is the unwrapped image resulting from (c).

\section{EXPERIMENTATION AND ANALYSIS}

To visually examine and benchmark the pose estimation and unwrapping results, the pose estimation and unwrapping techniques were verified via conducting two sets of empirical lab experiments. The first used a test card image; and the second used a representative pipe sample containing manufactured defects. The test card experiment is a demonstration of a higher feature space, representing a feature rich section of pipe such as that with extensive flaws. The unique and clear to interpret patterns also make it more practical to visually judge the performance of the process. The second sample used, an aluminum pipe with holes in a cross pattern is more representative of that which would be encountered in a practical inspection, lacking in significant features but with defects of a specific size and location to enable further verification of the process.

\section{A. Test Card Experiment}

The experiments were conducted with pipe lengths of 600 $\mathrm{mm}$. The measurements performed on the $83 \mathrm{~mm}$ pipe were conducted with a texture applied to the inner wall of the pipe; this texture is depicted in Fig. 9, this standard test pattern was chosen to clearly demonstrate the merits of our unwrapping technique.

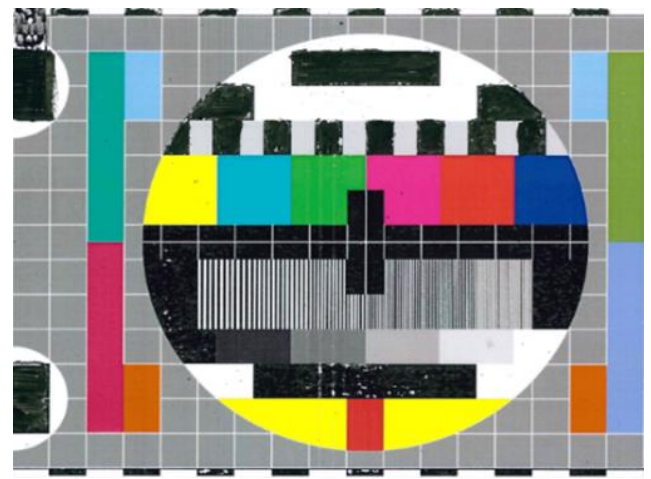

Fig. 9 The ground truth texture that was placed inside the pipe [27]

To remove the picture of the conical mirror assembly from the center of the images, a binary mask (as presented in Fig. 10) was applied to the images before unwrapping. This is necessary for the stitching phase, as it eliminates the fixed features that otherwise would exist in each frame and at the same location making image registration challenging. Fig. 10 gives an example of an image captured with a relative offset of $\alpha=3^{\circ}$, $\beta=-6^{\circ}, x=-14 \mathrm{~mm}, y=-18 \mathrm{~mm}$. Fig. 10 (a) and (b) show one pair of such images in the $65 \mathrm{~mm}$ diameter pipe. Assuming a centralized camera would produce image Fig. 10(c). Unwrapping with the corrected pose produces the unwrapped image Fig. 10 (d). 


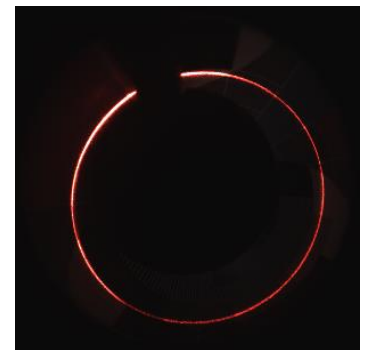

(a)

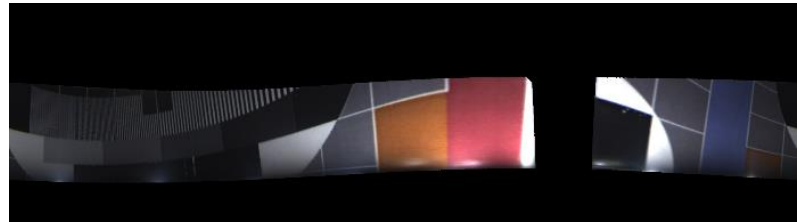

(c)

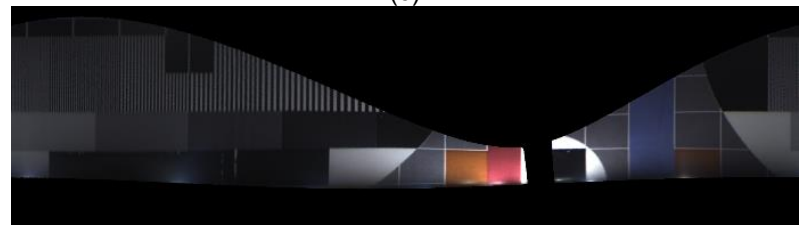

(d)

Fig. 10 An image pair consists of a dark image (a) and a bright image (b) from the same section of the pipe with the binary mask (green) applied before unwrapping. (d) unwrapped image assuming the camera was centralized $\left(\alpha=0^{\circ}, \beta=0^{\circ}, x=0 \mathrm{~mm}, y=0 \mathrm{~mm}\right)$ (e) unwrapped image using the corrected pose $\left(\alpha=3^{\circ}, \beta=-6^{\circ}, x=-14 \mathrm{~mm}, y=\right.$ $-18 \mathrm{~mm}$ )

Fig. 11 depicts the image stitching that are created by stitching 20 images captured along the length of the $83 \mathrm{~mm}$ ID pipe (as presented in Fig. 12) with the standard test pattern lining. Fig. 11(b) depicts the mosaic acquired from the same dataset using post-inspection centralization, it is shown to be less warped than the results acquired without any means of centralization, as Fig. 11(a).

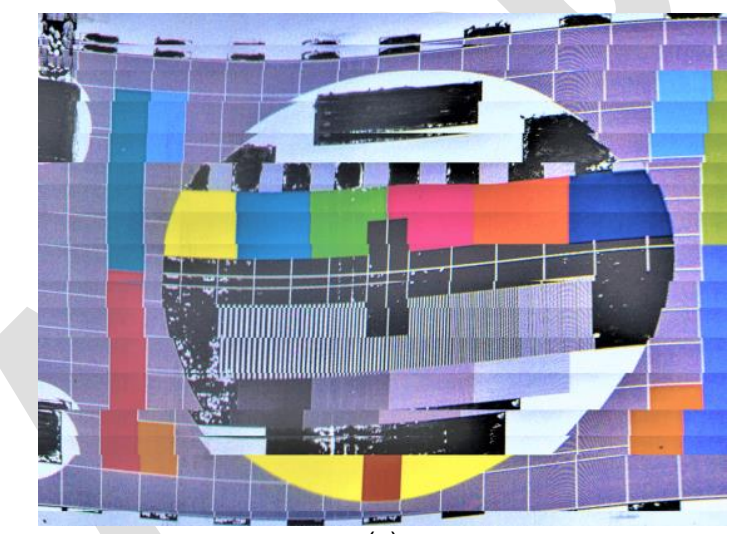

(a)

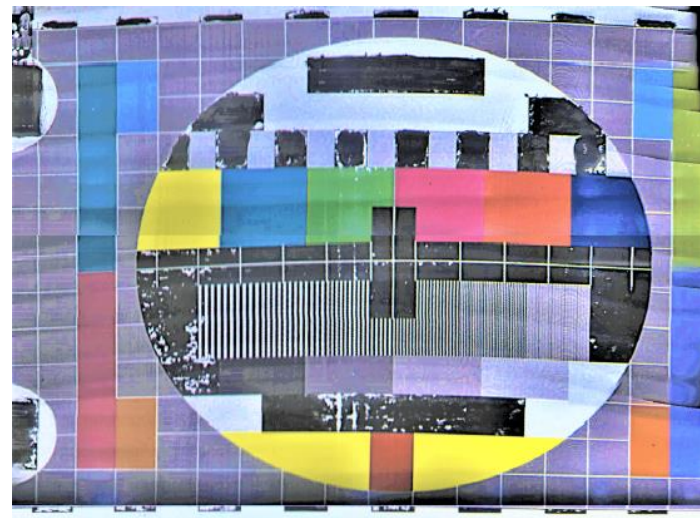

(b)

Fig. 11 Image mosaic created from stitching 20 unwrapped images of $83 \mathrm{~mm}$ diameter pipe sections with texture. (a) without centralization (b) with post-inspection centralization

An open source metric function, evaluated within the MATLAB data processing software environment, was utilized to quantify the degree of result image quality. This metric described by Zhou et al. [28] is based on a percentage similarity comparison between the original image (Fig. 9) and image after processing (Fig. 11). Higher metric index implies that the image had more matching features and is thus indicative of a better reconstructed image. The index of the image without centralization (Fig. 11(a)) is 0.3022 , whereas the index of the image with the post-inspection centralization (Fig. 11 (b)) is 0.3502 .

\section{B. Representative Defect Sample Experiment}

To provide a more realistic sample containing defects in known locations with standard sizing a test piece comprising of seven holes with equal diameters and distance from each other was manufactured from a section of aluminum pipe with an internal diameter of $65 \mathrm{~mm}$. Holes were drilled in a crossshaped pattern allowing verification of the method's accuracy in a practical inspection scenario, as shown in Fig. 12.

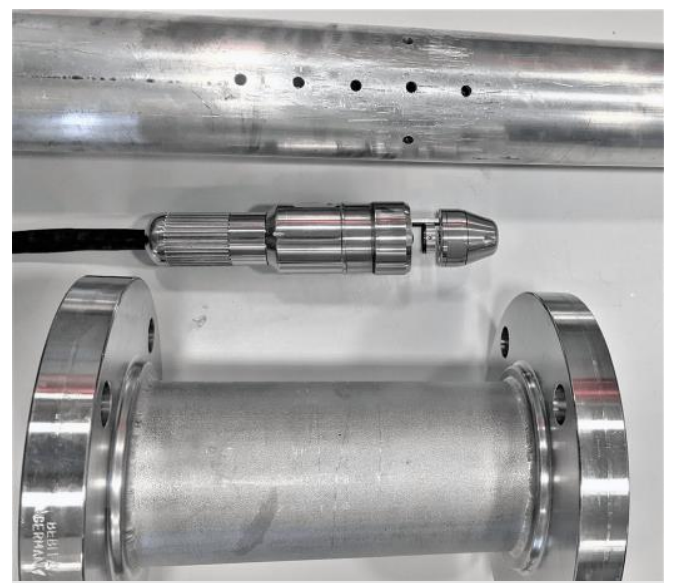

Fig. 12 Experiment setup. From the top to the bottom: the $65 \mathrm{~mm}$ diameter pipe, the probe hardware utilized in the experiment and the 83 $\mathrm{mm}$ diameter pipe. 


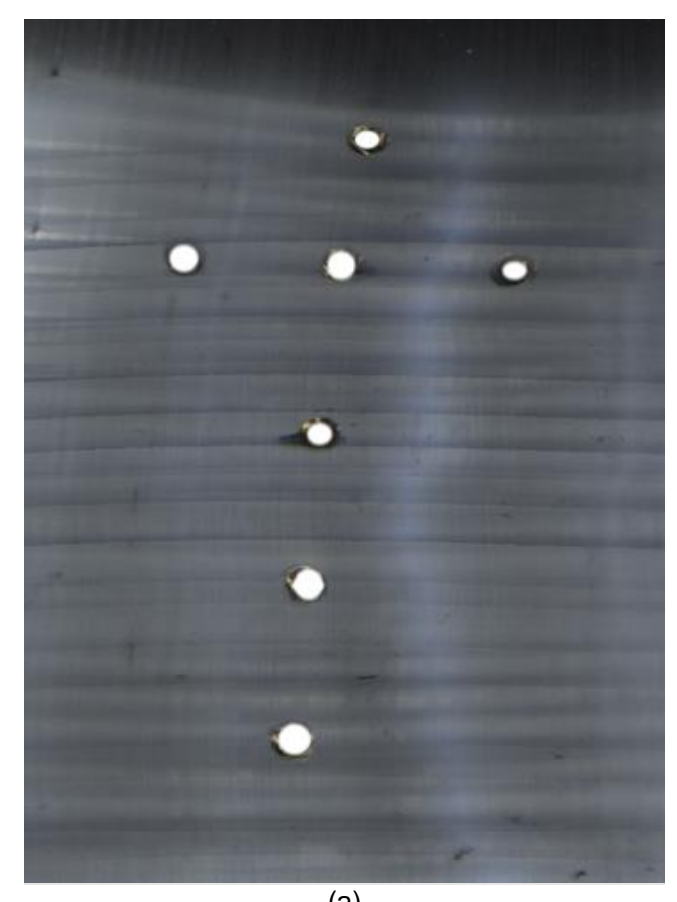

(a)

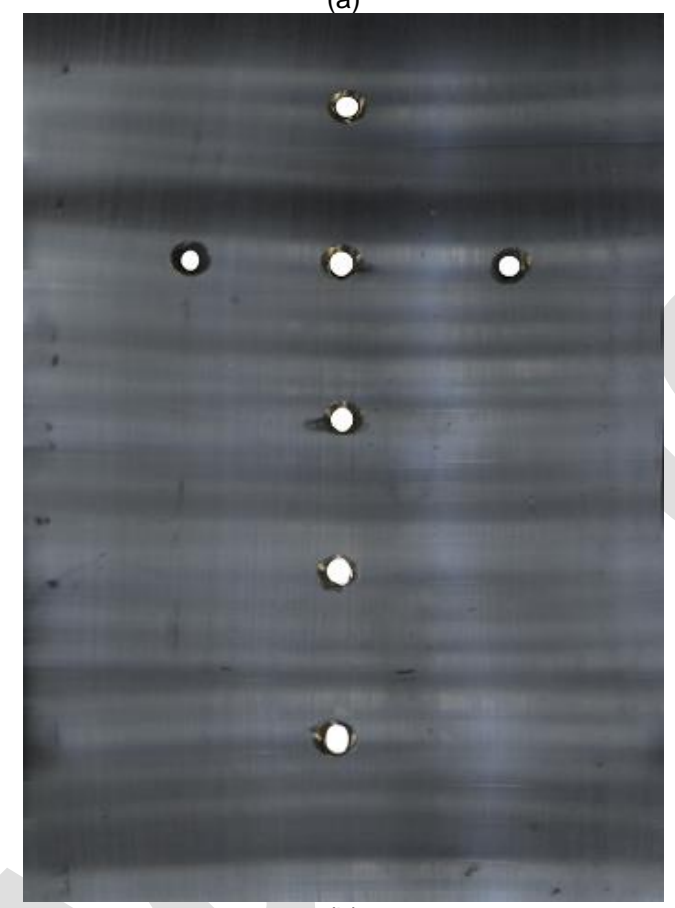

(b)

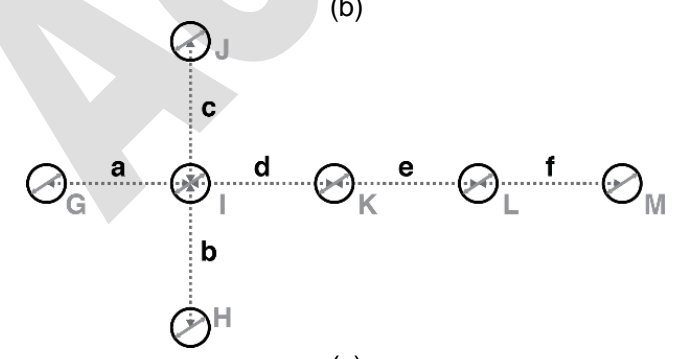

(c)

Fig. 13 Image mosaic created from stitching 32 unwrapped images of $65 \mathrm{~mm}$ diameter pipe sections. (a) without centralization (b) with post-inspection centralization (c) reference dimensions
Table 2 Measured distances (a-f) and diameters (G-M) from Fig. 13. (unit: $\mathrm{mm}$ )

\begin{tabular}{|c|c|c|c|}
\hline & Reference & $\begin{array}{c}\text { Synthetic } \\
\text { Centralization }\end{array}$ & $\begin{array}{c}\text { No } \\
\text { Centralization } \\
\end{array}$ \\
\hline $\mathbf{a}$ & \multirow{7}{*}{24} & 24.24 & 24.10 \\
\hline b & & 25.63 & 33.27 \\
\hline c & & 23.35 & 30.51 \\
\hline d & & 24.29 & 33.36 \\
\hline $\mathbf{e}$ & & 23.14 & 30.07 \\
\hline f & & 25.98 & 30.80 \\
\hline Average & & 24.43 & 30.35 \\
\hline $\begin{array}{l}\text { Standard } \\
\text { Deviation }\end{array}$ & & 1.06 & 3.08 \\
\hline G & \multirow{8}{*}{6} & 6.49 & 4.23 \\
\hline H & & 6.57 & 6.13 \\
\hline I & & 6.27 & 6.42 \\
\hline $\mathbf{J}$ & & 5.62 & 3.79 \\
\hline $\mathbf{K}$ & & 5.91 & 4.67 \\
\hline $\mathbf{L}$ & & 5.11 & 6.42 \\
\hline M & & 5.84 & 6.57 \\
\hline Average & & 5.97 & 5.46 \\
\hline $\begin{array}{l}\text { Standard } \\
\text { Deviation }\end{array}$ & & 0.48 & 1.09 \\
\hline
\end{tabular}

Fig. 13 demonstrates the image mosaic result acquired from the $65 \mathrm{~mm}$ diameter pipe. The image stitching result in Fig 13(a) is created using 32 images and it visualizes $160 \mathrm{~mm}$ of the pipe length, where equally distanced holes were drilled in a cross pattern inside the pipe. The reference hole diameter and distances between two holes are $6 \mathrm{~mm}$ and $24 \mathrm{~mm}$, respectively. Using the object space resolution $0.146 \mathrm{~mm} /$ pixels, the distances between two holes measured from Fig. 13(a) is on average $24.43 \mathrm{~mm}$ ( $1.8 \%$ error $)$ with the standard deviation of $1.06 \mathrm{~mm}(4.4 \%)$. In contrast, the distances measured from Fig. 13 (b) is $30.35 \mathrm{~mm}$, gave the mean error of $6.35 \mathrm{~mm}(26.4 \%)$ with a standard deviation of $3.08 \mathrm{~mm}(12.83 \%)$. The data are summarized in Table 2. Similarly, the average error and standard deviation of the hole's diameter were reduced from $0.54 \mathrm{~mm}$ to $0.03 \mathrm{~mm}$ and 1.09 to $0.48 \mathrm{~mm}$, respectively.

\section{CONCLUSION}

This article demonstrated a novel post-inspection centralization method for visual inspection where options for mechanical centralization is limited. This method is comprised of active pose estimation and unwrapping algorithms. The active pose estimation provided the camera pose to the unwrapping algorithm, which then corrected the images.

It was indicated that the pose estimation relies on the changes of $\Delta P$ due to this value being the main contributor to the optimization, this $\Delta P$ value is influenced by the camera model and pipe diameter. Fig.7 depicted that the maximum $\Delta P$ value is minimal when the probe is positioned with small rotational and translational deviations from the center. The impact of such negligible rotation and translations are not visually perceivable, and the deviation of the camera from center can be ignored.

An active pose estimation technique using a laser-profiler made the calculation of pose independent to the nature and quality of the image (SfM is heavily dependent on these [29], [30]). The experiments verified that the camera pose could be retrieved $\pm 0.5 \mathrm{~mm}$ and $\pm 0.5^{\circ}$ error margin. As depicted in Fig. 11 , the visual distortion caused by this level of pose estimation error is negligible. Real world pipes have a degree of ovality 
(typically up to $1.5 \%$ of the nominal OD [31]). The current approach will confuse such ovality with a slight rotation of the probe head, leading to an error. Furthermore, if the pipe is heavily corroded or deformed, the algorithm will struggle to accurately estimate the probe pose and unwrap correctly. The method currently only works for straight sections of pipes as bends will lead to additional warping. This work made use of an existing probe with a laser ring projector for pose estimation, future work could optimize the laser projection, so it does not fall on a plane, making it easier to estimate probe's relative poses.

In summary, the paper presented a post-inspection centralization method, achieved comparable results using a mechanically centralized probe. This resulted in a compact and minimalistic probe design which was utilized for the inspection of small-bore pipes. Using this method, the error of the distance measurement from an industrial asset is $1.8 \%$. Thus, the resulted pipe stitching can be used to inspect and localize the pipe defects, making pipe RVI applicable and more reliable for pipe inspections.

\section{REFERENCES}

[1] J. C. Bugler, "Pipelines safety regulations," Inst. Chem. Eng. Symp. Ser., no. 139, pp. 525-535, 1995.

[2] E. Hong, R. Katz, B. Hufnagel, and J. Agapiou, "Optical method for inspecting surface defects inside a small bore," Meas. Sci. Technol., vol. 21, no. 1, 2010.

[3] M. Bibuli, M. Caccia, and L. Lapierre, "Robotic Devices for Water Main In-Pipe Inspection: A Survey," IFAC Proc. Vol., vol. 7, no. PART 1, pp. 81-86, 2007.

[4] Eddyfi, "Versatrax 150 Pipe Inspection Crawler," 2020. [Online]. Available: https://eddyfi.com/en/product/versatrax-150-pipeinspection-crawler. [Accessed: 21-Jul-2020].

[5] Y. S. Kwon and B. J. Yi, "Design and motion planning of a twomodule collaborative indoor pipeline inspection robot," IEEE Trans. Robot., vol. 28, no. 3, pp. 681-696, 2012.

[6] P. Hansen, H. Alismail, P. Rander, and B. Browning, "Visual mapping for natural gas pipe inspection," Int. J. Rob. Res., vol. 34, no. 4-5, pp. 532-538, 2015.

[7] J. Gang, "Structured-light 3D surface imaging: a tutorial," Adv. Opt. Photonics, vol. 3, pp. 128-160, 2011.

[8] J. L. Schonberger and J. M. Frahm, "Structure-from-Motion Revisited," Proc. IEEE Comput. Soc. Conf. Comput. Vis. Pattern Recognit., vol. 2016-Decem, pp. 4104-4113, 2016.

[9] O. Duran, K. Althoefer, and L. D. Seneviratne, "Automated pipe defect detection and categorization using caniera/laser-based profiler and artificial neural network," IEEE Trans. Autom. Sci. Eng., vol. 4, no. 1, pp. 118-126, 2007.

[10] T. Wu, S. Lu, and Y. Tang, "An in-pipe internal defects inspection system based on the active stereo omnidirectional vision sensor," 2015 12th Int. Conf. Fuzzy Syst. Knowl. Discov. FSKD 2015, pp. 2637-2641, 2016.

[11] P. V. Unnikrishnan, B. Thornton, T. Ura, and Y. Nose, "A conical laser light-sectioning method for navigation of autonomous underwater vehicles for internal inspection of pipelines," Ocean. '09 IEEE Bremen Balanc. Technol. with Futur. Needs, 2009.

[12] A. Dehghan Tezerjani, M. Mehrandezh, and R. Paranjape, "4-DOF pose estimation of a pipe crawling robot using a Collimated Laser, a conic mirror, and a fish-eye camera," Proc. IEEE Southwest Symp. Image Anal. Interpret., pp. 45-48, 2014.

[13] J. A. Nelder and R. Mead, "A Simplex Method for Function Minimization," Comput. J., vol. 7, no. 4, pp. 308-313, 1965.

[14] G. West, P. Murray, S. Marshall, and S. McArthur, "Improved visual inspection of advanced gas-cooled reactor fuel channels," Int. J. Progn. Heal. Manag., vol. 6, no. SP3, pp. 1-11, 2015.

[15] W. Jackson, G. Dobie, C. MacLeod, G. West, C. Mineo, and L. McDonald, "Error Analysis and Calibration for a Novel Pipe Profiling Tool,” IEEE Sens. J., vol. 20, no. 7, pp. 3545-3555, 2020.
[16] A. V. Reyes-Acosta, I. Lopez-Juarez, R. Osorio-Comparan, and G. Lefranc, "3D pipe reconstruction employing video information from mobile robots," Appl. Soft Comput. J., vol. 75, pp. 562-574, 2019.

[17] MathWorks, "Optimization Toolbox," 2020.

[18] D.G.Lowe, "Distinctive image features from scale-invariant keypoints," Int. J. Comput. Vis., vol. 60, no. 2, pp. 91-110, 2004.

[19] J. Choi, C. M. Yeum, S. J. Dyke, and M. R. Jahanshahi, "Computeraided approach for rapid post-event visual evaluation of a building Façade," Sensors (Switzerland), vol. 18, no. 9, 2018.

[20] Eddyfi, "Eddyfi Spectrum 45," 2020. [Online]. Available: https://www.eddyfi.com/en/product/spectrum-45. [Accessed: 21Jul-2020].

[21] Baker Hughes, "Baker Hughes Video Borescopes," 2020. [Online]. Available: https://www.bakerhughesds.com/inspection-ndt/remotevisual-inspection/video-borescopes. [Accessed: 21-Jul-2020].

[22] D. Scaramuzza, A. Martinelli, and R. Siegwart, "A flexible technique for accurate omnidirectional camera calibration and structure from motion," Proc. Fourth IEEE Int. Conf. Comput. Vis. Syst. ICVS'06, vol. 2006, p. 45, 2006.

[23] Jean-Yves Bouguet, "Camera Calibration Toolbox for Matlab," 2015. .

[24] Mathworks, "Fisheye Calibration Basics," 2020.

[25] H. Araujo and J. M. Dias, "An introduction to the log-polar mapping [image sampling]," pp. 139-144, 2002.

[26] C. Fehn and H. Hhi, "Depth-Image-Based Rendering ( DIBR ), Compression and Transmission for a New Approach on 3D-TV," Proc. SPIE, vol. 5291, pp. 93-104, 2004.

[27] J. Kannala and S. S. Brandt, "A generic camera model and calibration method for conventional, wide-angle, and fish-eye lenses," IEEE Trans. Pattern Anal. Mach. Intell., vol. 28, no. 8, pp. 1335-1340, 2006.

[28] Philips, "Philips PM5544.” 2020.

[29] Z. W, B. A. C, S. H. R, and S. E. P, "Image Qualifty Assessment: From Error Visibility to Structural Similarity," IEEE Trans. Image Process., vol. 13, no. 4, pp. 600-612, 2004.

[30] D. Zhang, R. Watson, G. Dobie, C. MacLeod, A. Khan, and G. Pierce, "Quantifying impacts on remote photogrammetric inspection using unmanned aerial vehicles," Eng. Struct., vol. 209, p. 109940, Apr. 2020.

[31] S. Bianco, G. Ciocca, and D. Marelli, "Evaluating the performance of structure from motion pipelines," J. Imaging, vol. 4, no. 8, 2018. ASME, "ASTM-A999 | Standard Specification for General Requirements for Alloy and Stainless Steel Pipe,” 2018. 